\title{
The measuring of temperature fields in two and three dimensional airflows using an infrared camera
}

by M. Pešek and M. Pavelek*

*Dept. of Thermodynamics and Environmental Engineering, Brno University of Technology, Technická 2896/2, 61669 Brno, Czech Republic, m.pesek@email.cz

\begin{abstract}
This article deals with the measuring of temperature fields in the two and three dimensional airstreams using an infrared camera. This method is based on the visualization of temperature fields on an auxiliary material (for two dimensional airflow) or on measuring targets in measuring net (for three dimensional airflow) which is inserted into the research area. The method for measuring temperature fields in the air by an infrared camera can be used in many various applications such as air-heating and air-conditioning.
\end{abstract}

\section{Introduction}

The infrared (IR) camera is a very effective device for a noncontact measurement of temperature fields in many scientific disciplines [6]. This measuring method provides a visual image records and the information for deeper knowledge of thermal states and processes in the research object. The thermography is being used mainly for determining surface temperatures, but this article tries to extend the possibilities of monitoring and the measurement of temperature fields in the air.

Immediate knowledge of the distribution of temperature field in the non-isothermal airflow can allow to quickly identify a possible problem in the design of air conditioning or hot-air heating. The visualization of temperature fields in the air can also detect the spatial and time contexts of monitored processes.

\section{Principle of temperature field measurement using an infrared camera}

It is well known that the air is a transparent material in infrared radiation, it is impossible to make the air temperature visible directly by the infrared camera. For visualization the air temperatures in non-isothermal air flows, an auxiliary material, which has to be inserted into airstreams, is necessary to be used [2]. The hot airflow produces the convective heat transfer to the surface of auxiliary material that changes the surface temperature which is measured trough surface temperature by the infrared camera. The infrared camera is ordinary used for non-contact measurement, but this method for measuring temperature field is a contact method. Two measuring methods are recognized. For measuring temperature fields in two dimensional airflow, a spacious sheet of auxiliary material is suitable be used and for three dimensional airflow the measuring net is needed to use.

For application of this method, the suitable materials were needed to choose. These materials must especially have a large emissivity value and the quick response to the temperature difference (small time constant) [4]. The auxiliary material of known emissivity is inserted to the airstream. It is necessary to put the infrared camera appropriately to the view of the research airstream. The IR camera has to be positioned at the right angle to the auxiliary material. After that it is necessity for measuring and evaluation of the auxiliary material surface temperature to know the radiation temperatures of surroundings areas which have the effect on auxiliary material and to define the middle radiation temperature. If there is some unwanted source of heat, it has to be removed or eliminated it. The infrared measuring is done in infrared camera stable state (the minimum is after 15 minutes) and in a temperature field stable state (three to fifth time constants [4]) and after the auto calibration of the camera (the calibration makes promises of the accurate measuring). After that the emissivity value and the middle radiation temperature are set to the IR camera software. The temperature fields are shown directly on the display of the infrared camera or on the display of connecting notebook in Irbis Online program.

The method of measuring temperature fields in the air by infrared camera is very good applicable for heat sources (or cold sources) where the velocity and the temperature values are larger than in the still microclimate.

\section{Measuring of temperature fields in 2D airflows}

The visualization of temperature fields in the air by the thermovision on a spacious sheet of auxiliary material is suitable only for measuring temperature fields in two dimensional airflows. The measuring temperature fields in $2 \mathrm{D}$ airflows it can be imagined the measurement where the compact auxiliary material (compact sheet) is parallel with streamlines. 


\subsection{Device description for measuring temperature fields in 2D airflows}

In Fig. 1 the device shows the temperature field on an auxiliary material $A M$ from various heat or cold sources $S$ using the infrared camera VarioCam IRC linked to notebook $N$. At the same time the radiating temperature is measured in the measuring area by the infrared thermometer Testo IRT. The sheet of an auxiliary material is attached to the holder $H$ to the height-adjustable tripod-stand $T$.
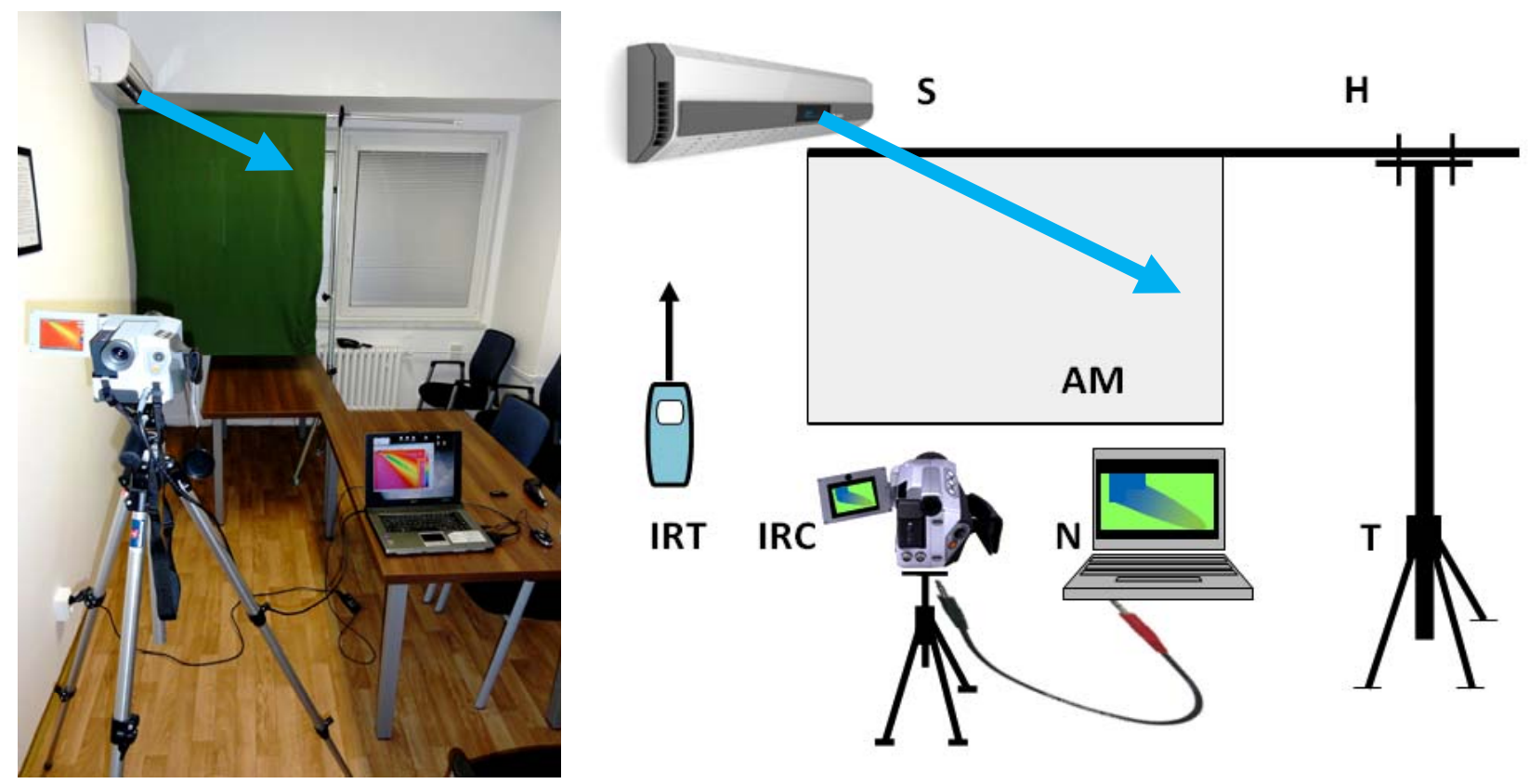

Fig. 1. The stand for measuring the air temperature by an infrared camera (photo and schema) IRC - Infrared Camera, N - Notebook, AM - Auxiliary Material, T - Tripod, H - Holder, IRT - Radiating Thermometer, S - Source of Non-Isothermal Airflow

The surface of auxiliary material must be fully plane in order to eliminate measurement inaccuracy and its sizes are proportional to the temperature field visualized. As an auxiliary material the planar office paper and the cotton textile with high the emissivity value were chosen [4]. The office paper is suitable for an accurate measuring, but it is impractical to store. The cotton textile is easy to store therefore it is applicable for mobile equipment. Both materials have a no-reflection surface, low heat capacity, low heat conductivity and minimal thickness. These quantities have an influence to dynamic properties of this measuring method and they are expressed by the time constant. The device is easy to move and the response of measuring air temperature is practically immediate.
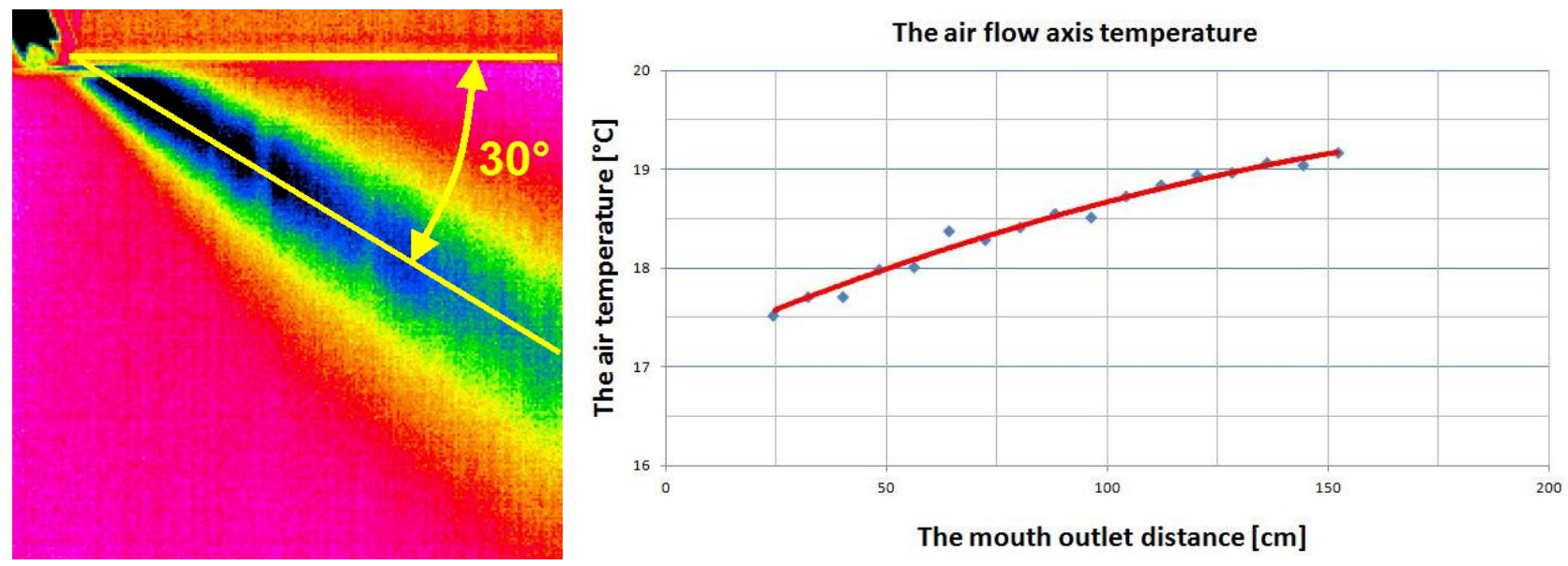

Fig. 2. The thermogram of measured temperature field in $2 D$ airflow and the dependence temperature increase in the axis of cold airstream 


\subsection{Example of measuring temperature fields in $2 \mathrm{D}$ airflows}

Fig. 2 shows the two dimensional temperature field in the cold airstream from the cooling equipment. The cooling equipment creates the uniform airstream where is possible to use the spacious sheet of auxiliary material. In the figure the dependence of temperature increase in axis of cold airstream is demonstrated. In showed case the axis of airstream is tilted about $-30^{\circ}$ from the horizontal plane. The origin of coordinates is positioned in the mouth outlet.

\section{Measuring of temperature field in 3D airflows}

The visualization of temperature fields in the air by the thermovision on measuring targets (Fig. 3) of known properties is especially suitable for measuring temperature fields in three dimensional airflows. In notion of measuring temperature fields in 3D airflows it can be imagined the measurement where the auxiliary material (compact sheet) is not parallel with streamlines, for example where a rotate circle airstream is generated from the ventilator, or alternatively more heat sources are used and so one.

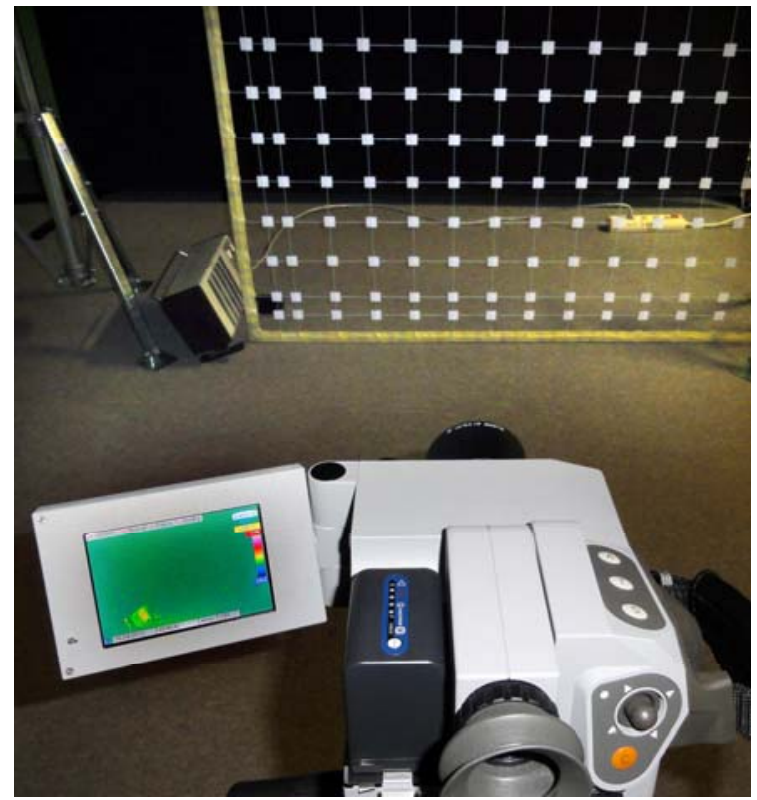

Fig. 3. The photography of the equipment from measuring of temperature fields in $3 D$ airflow with measuring targets by an infrared camera

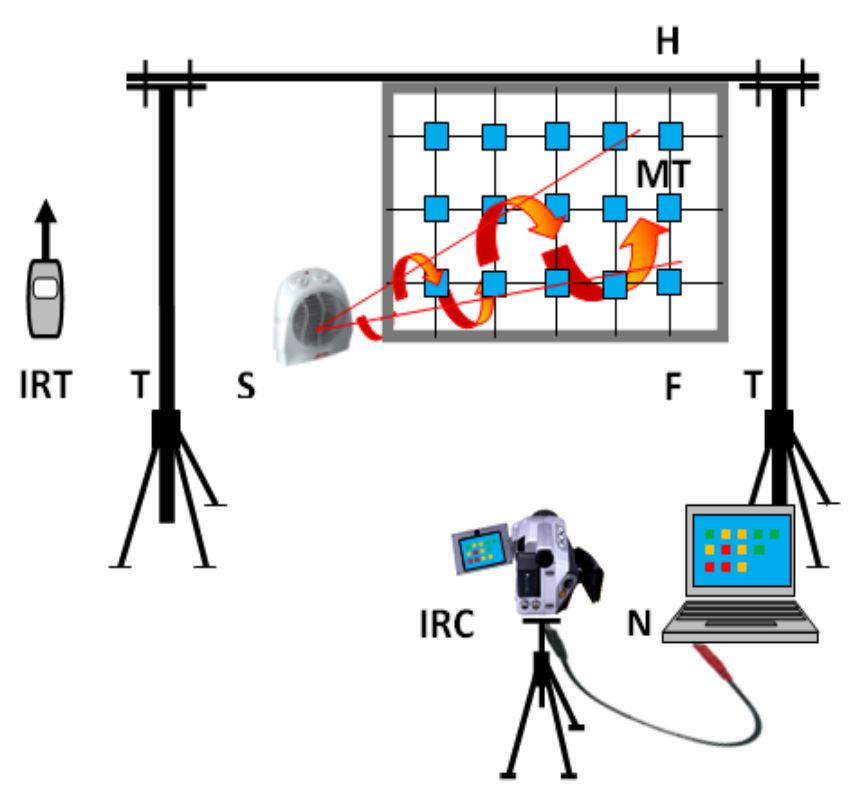

Fig. 4. The stand for measuring temperature fields in three dimensional airflow by the ventilator with a rotate circle airstream by an infrared camera

\subsection{Device description for measuring temperature fields in 3D airflows}

In Fig. 4 the schema of stand for measuring temperature fields in 3D airflow is shown. The equipment is a very similar to the equipment for measuring temperature fields in $2 \mathrm{D}$ airflow with the difference that the frame $F$ with measuring targets $M T$ replaces the compact sheet of auxiliary material $A M$. The targets are made from planar office paper with high emissivity value [4].

Fig. 5 shows the prototype of measuring net for usage of measuring temperature field in the cabin of small transport airplane EV-55. This frame with measuring net with target was built for the experiment in order to investigate how the measuring targets will respond to changing airflow temperatures [2].

Based on this prototype the new measuring frame with a better quality for a versatile usage was made (Fig. 6). The measuring frame is the weldment which was made of bars and its size is $1.520 \times 1.320 \mathrm{~mm}$. The material of this frame is an alloy AIMg3. Because the stiffness of this frame was not acceptable for measuring, the line both-sides weld was welded on the frame. To the eliminate reflectivity the frame with non-reflectivity tape was supplied. The upgrade measuring net is compounded of measuring targets of known properties. The targets are situated on the net with the exactly defined distance. All the measuring targets are situated on cords and targets and cords have together only $9.6 \%$ of area of all area in this measuring frame. The targets are made from the planar office paper with high emissivity value $(\varepsilon=0.96)$ and the cords are made from non-colour polypropylene (non-colours polypropylene cords have better properties compared to the coloured polypropylene cords).

The thermal expansivity of these cords is no more than $19 \%$ of their length in the temperature range from $20{ }^{\circ} \mathrm{C}$ to $90{ }^{\circ} \mathrm{C}$. At a higher temperature difference the cords extension would be too length and the measuring net would collapse. The temperature difference of $70 \mathrm{~K}$ corresponds to temperatures suitable for heating and cooling buildings and suitable for measuring temperature fields in still indoor microclimate [1]. 


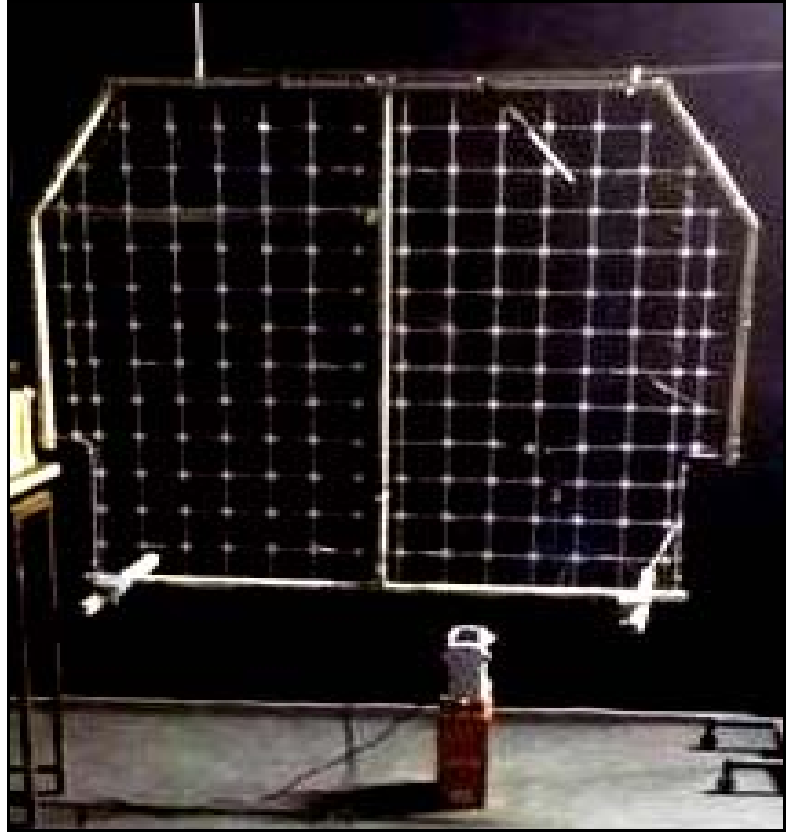

Fig. 5. The prototype photography of measuring temperature fields in $3 D$ airflows

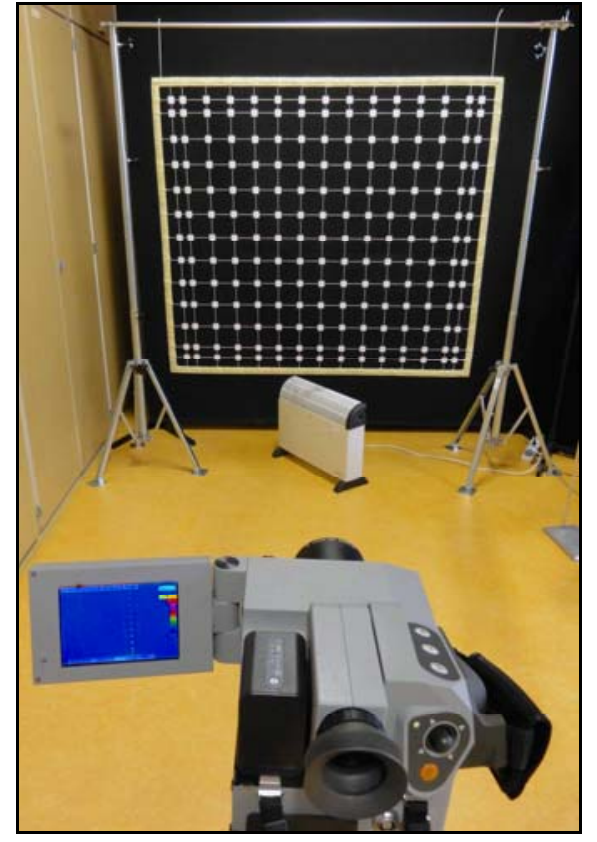

Fig. 6. The photography of measuring equipment for measuring 3D airflows with better quality

The sizes of measuring targets should be chosen such that on the thermogram their sizes are $5 \times 5$ pixels minimally. To guarantee this condition the measuring targets were suggested to be $26 \times 26 \mathrm{~mm}$ in order to visible the entire frame on the thermogram by using the VarioCam infrared camera from Jenoptic Corporation.

\subsection{Example of measuring temperature fields in 3D airflows}

For the following experiment the hot-air rotation ventilator was chosen. The rotation airstream from this ventilator is generated, and therefore it is necessary to use an auxiliary material for the 3D airstream visibility where temperature field is possible to measure. The original temperature field visualization of this ventilator is shown in Fig. 7. The area between the measuring targets must be software adapted (Fig. 8) to produce a complete thermogram of temperature fields in 3D airflow.

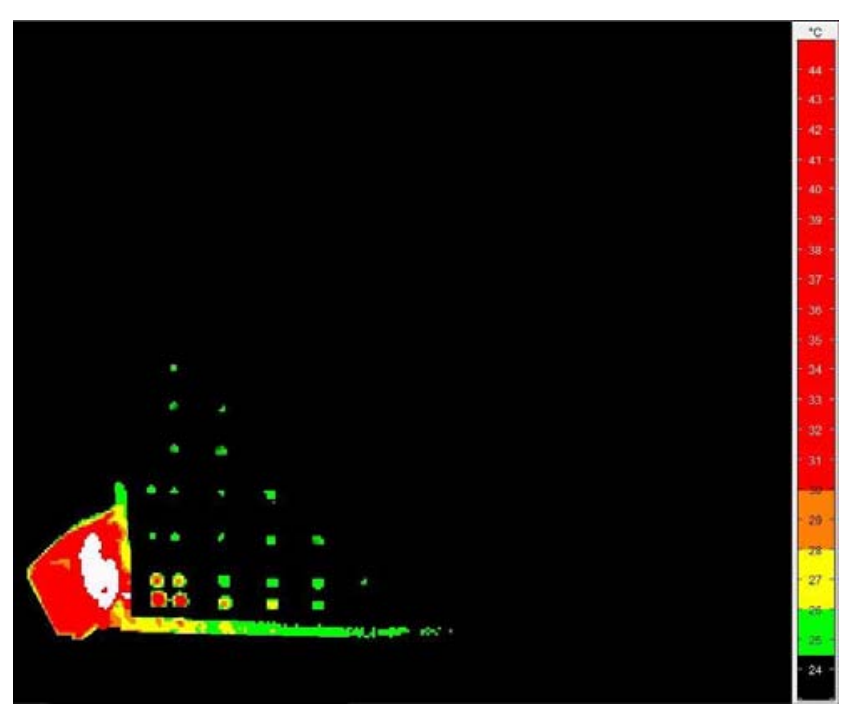

Fig. 7. The visualization of temperature field in $3 D$ airflow using measuring targets and the infrared camera

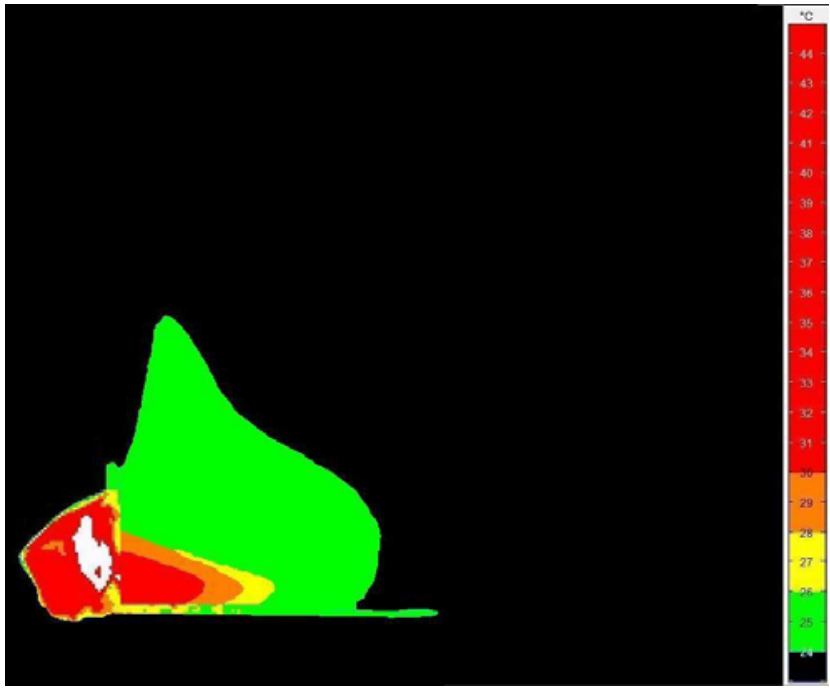

Fig. 8. The visualization of temperature field in $3 D$ airflow using measuring targets and the infrared camera after software adjustment 


\section{The method comparison of temperature fields measuring in 2D and 3D airflows}

The main advantage of measuring targets with respect to measuring sheet is the small influence of the airstream by own measuring. The airstream can pass through the net and it is possible to measure more correct air temperatures. In case of compact sheet of auxiliary material the airstream, which is not parallel with sheet of material, impacts to the obstacle, it changes its direction and devalues the measuring results. The second main advantage is that measuring net can easily define the scale of view even when the wide-angle lens is used which makes the thermogram distorted, especially at corners of thermogram.

On the other side the main disadvantage is that the evaluation with using the net with targets is more complicated in a comparison to using compact sheet of auxiliary material. It is necessary to define the area around the measuring targets and on this area the maximum temperature (by heat sources) or the minimum temperature (by cold sources) must be determined. These temperatures are possible to be taken as temperatures of airflows in these points. The obtained thermogram does not create a complete picture (a complete temperature field) therefore the temperature field measurement on targets points to complete the thermogram must be completed after the own measurement (Fig. 8). This operation is very time-consuming. The next disadvantage is that the measuring net production for specific applications is much more complicated.

On the next picture (Fig. 9) the difference of measuring 3D rotation airstream on the device with an auxiliary material for temperature field in 2D airflow is shown. The temperature field in $3 \mathrm{D}$ airflows which is visualized is a very different on the compact sheet of textile cotton compared to usage of measuring targets (Fig. 8) because the compact sheet restricts the airflow.

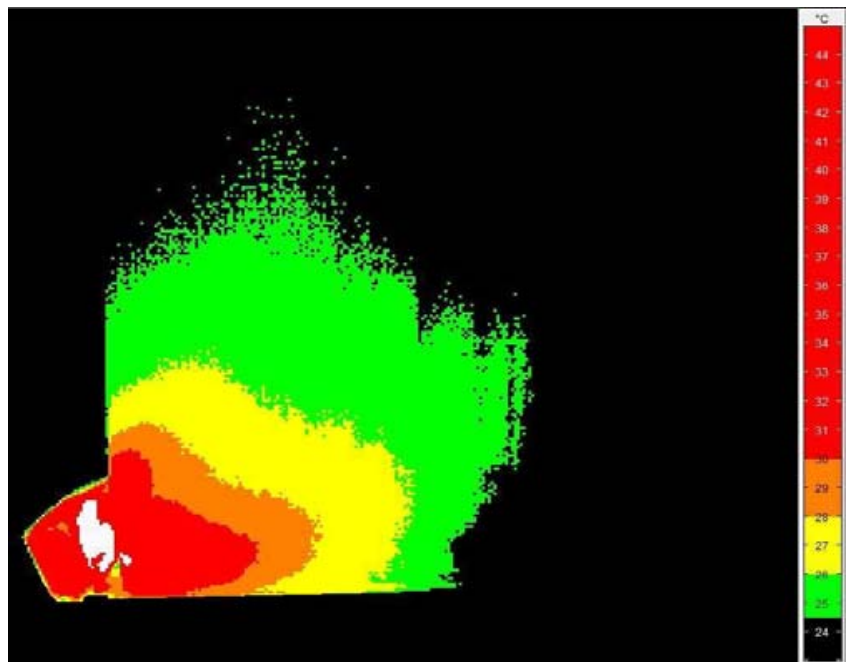

Fig. 9 The wrong visualization of temperature field in $3 D$ airflow using a compact sheet of auxiliary material and the infrared camera

\section{Discussion}

The air is transparent material and it is necessary to use the auxiliary material for measuring airstreams. The measurement results show that the usage of the temperature visualization in the air with using auxiliary materials is illustrative and provides qualitative information immediately. When the correct emissivity value of the auxiliary material and the radiation temperatures are entered, the air temperature can be measured with a measurement uncertainty of the absolute temperature $2 \mathrm{~K}$ [4]. The uncertainty of temperature differences measurement is approximately $0.1 \mathrm{~K}$. The question is whether this uncertainty for particular application of thermal comfort and relevant standards is sufficient [1]. The results also illustrate that measuring network for temperature fields in 3D airflows is suitable well designed and the auxiliary material for the measurement of temperature fields in $2 \mathrm{D}$ airflows is suitable. It has been shown that when a compact of the auxiliary materials for the measurement of temperature field in 3D airflows is used, then the air temperature measurement is distorted.

\section{Conclusion}

The measuring of temperature fields in the air by infrared camera is one of the possible methods how to measure the temperature fields in the air. In opposite to other methods the measuring by the infrared camera is quick and allows the visualization of the non-isothermal air streams with using a simple principle. The usage of a spacious sheet of material is suitable for temperature fields in two dimensional airstream. For temperature fields in three dimensional airstreams the measuring net is needed to use. The proposed device is mobile and it can be used outside of the laboratory in various practical applications. 


\section{Acknowledgement}

The support of this research from Czech Science Foundation project no. GA 101/09/H050 "Research on energy efficient systems to reach indoor environment comfort", and the standard project FSI-S-11-6 "Human Centered Design" is gratefully acknowledged.

\section{REFERENCES}

[1] ISO 7730. Moderate thermal environments - Determination of the PMV and PPD indices and specification of the conditions for thermal comfort. 2005.

[2] FIŠER, J.; MLČÁK, R.; PAVELEK, M.; JíCHA, J. - Measurements of Temperature Patterns in Ventilated Spaces by Novel Measurement Method, Experimental Fluid Mechanics 2008, pp.36-41, ISBN 978-80-7372-417-7, (2008), Technical University of Liberec (Czech Republic)

[3] MINKINA, W., DUDZIK, S. - Infrared Thermography: Errors and Uncertainties. John Wiley and Sons, Częstochowa University of Technology, Poland 2009. ISBN 978-0-470-74718-6.

[4] PEŠEK M., PAVELEK M. - Determination of needed parameters for measuring temperature fields in the air by thermography. Experimental Fluid Mechanics, vol. 2, pp. 888-892, Technical University of Liberec, Jičín (Czech Republic), 2011.

[5] VOLLMER, M., MÖLLMANN, K. - P. - Infrared Thermal Imaging: Fundamentals, Research and Applications. WILEY-VCH Verlag GmbH \& Co. KGaA, Germany 2010. ISBN 978-3-527-40717-0.

[6] WILLIAMS, T. - L. - Thermal Imaging Cameras: Characteristics and Performance. CRC Press, Taylor \& Francis Group, Bromley (England) 2009. ISBN 978-1-4200-7185-6. 